\title{
Microstructure and mechanical properties of 304L steel fabricated by arc additive manufacturing
}

\author{
Lei Ji, Jiping Lu, Changmeng Liüa, Chenchen Jing, Hongli Fan, Shuyuan Ma \\ School of Mechanical Engineering, Beijing Institute of Technology, Beijing, Zhongguancun south Street, 100081, China
}

\begin{abstract}
For 304L large structural parts used in nuclear power, it is hard and costly to fabricate and machine traditionally. Wire arc additive manufacturing (WAAM) has low cost and high material utilization, which provides an efficient way to fabricate the large structural parts. So in this study, WAAM is used to fabricate the parts of $304 \mathrm{~L}$ stainless steel. Through the tensile test and metallographic analysis, the mechanical properties and microstructure of the 304L stainless steel fabricated by WAAM were explored. The results indicate that with the layers depositing, the cooling rate becomes slower, the dendrites become thicker and the morphology becomes more stable. Due to the existence of dendrites, the grain boundary strengthening effect is different between the transverse direction and longitudinal direction, and resulting in anisotropy of mechanical properties. However, the mechanical properties of the parts correspond to the forged piece, which lays the foundation for future applications.
\end{abstract}

\section{Introduction}

As a variant of traditional 304 stainless steel, 304L is a kind of austenitic stainless steel with pretty low carbon content, which is also known as ultra-low carbon stainless steel. Because of its good corrosion resistance, formability, excellent plasticity and toughness, the 304L metal products could perform well in high temperature conditions, the fabricating performance is excellent as well [1]. Especially, 304L stainless steel is a significant structural material in nuclear power equipment [2], also it is widely used in chemical industry, furniture decoration industry and medical industry. However, for the 304L large nuclear structure, the traditional manufacturing methods are limited by the long manufacturing cycle and high cost.

The emergence of the additive manufacturing provides a new way to fabricate the structure with 304L components. As a rapid prototyping technology, Additive Manufacturing (AM) is a promising manufacturing ways building objects layer by layer $[3,4]$. Based on the digital model, different metal materials or plastic and other adhesive materials could be fabricated by AM. Different with the traditional material removal processing technology, AM is a 'bottom - up' manufacturing method. Due to the innovation in the manufacturing process, it is considered to be 'an important production tool of the third industrial revolution'. Compared with the traditional manufacturing methods, The AM method can effectively save the processing time cost, reduce the amount of post-processing time, also can reduce the waste of materials and improve the utilization of materials [5].

\footnotetext{
a Corresponding author: liuchangmeng@bit.edu.cn
}

According to the different heat sources, the current existence of the AM method consists of laser melting deposition [6, 7], wire arc additive manufacturing [8, 9], electron beam additive manufacturing[10]. Many researches have been done on the stainless steel using the AM method. Oguzhan Yilmaz et al. [11] investigated the influence of different process parameters on the macrostructure and microstructure of the deposited AISI 308LSi parts. They clarified and compared the morphology and microstructure of the parts manufactured by traditional method and pulsed current TIG-SMD methods. K Abd-Elghany et al. [12] evaluated the different properties of products fabricated by selective laser melting (SLM). They confirmed that it's possible to fabricate the parts with fine details with the lowcost 304L powder used. Zhuqing Wang et al. [13] investigated the effect of processing parameters on the mechanical properties of AISI304L stainless steel fabricated by laser-based directed energy deposition AM. They found that the tensile samples with different fabricating directions and different linear heat inputs have various mechanical strength and elongation. Beth E Carroll et al. [14] investigated the anisotropic mechanical properties of a Ti6Al-4V part fabricated using a directed energy deposition (DED) process. They attributed the high ductility to the prevention of lack of fusion porosity in the finished component.

However, a comprehensive study that describes the 304L stainless steel fabricated by WAAM remains a gap in the existing research in the WAAM field. Compared with other AM methods, WAAM has low cost, high material utilization and high deposition rate [15]. It is suitable for large structural parts of nuclear power. 


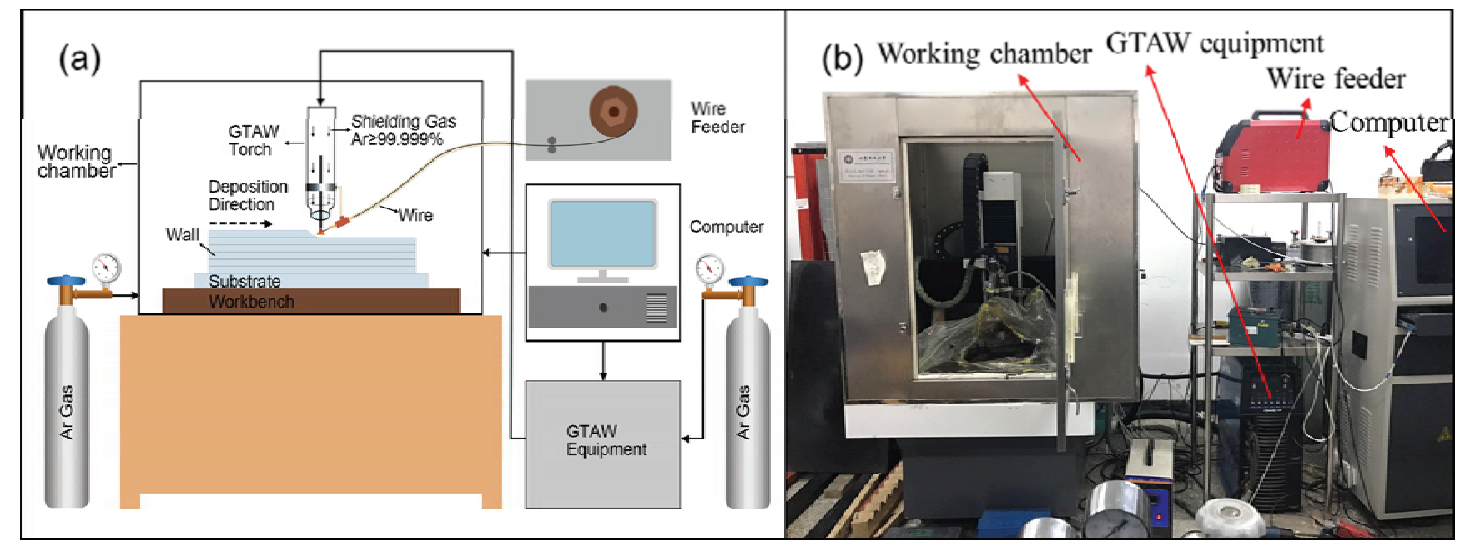

Figure 1. (a) Schematic of the experimental setup developed and (b) overview of WAAM system
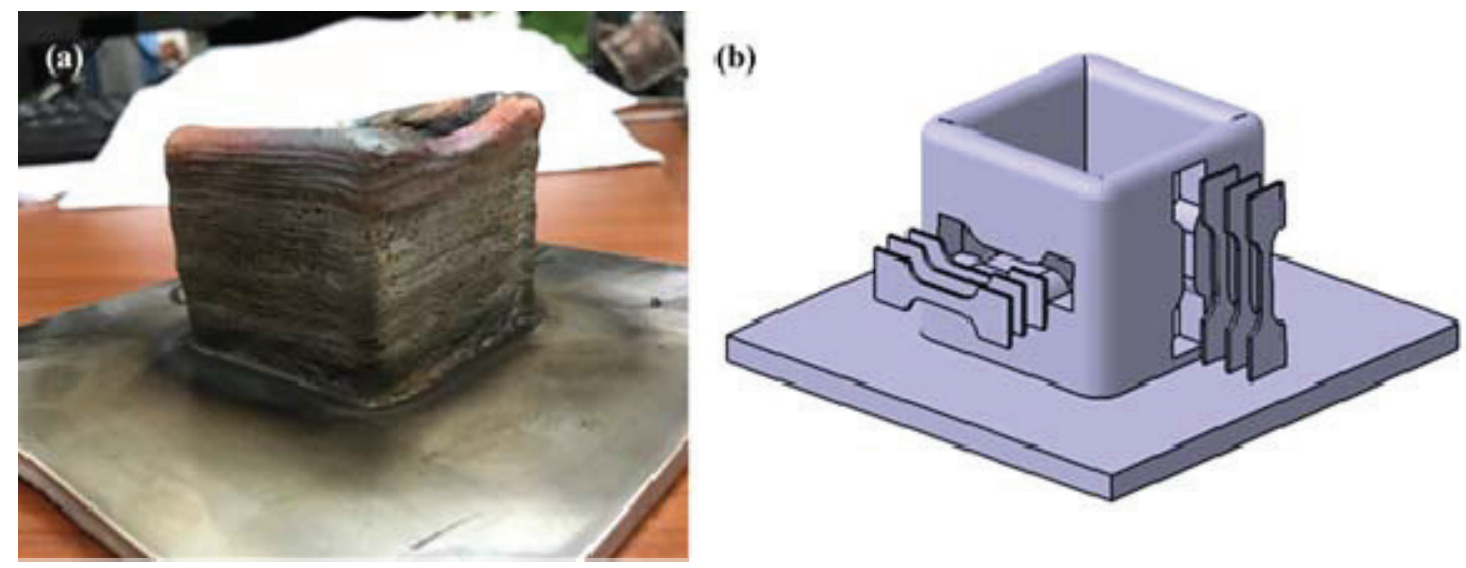

Figure 2. (a) The actual part fabricated by WAAM in this study and (b) schematic showing how the tensile specimens were extracted from the part.

So this study investigated the morphology, microstructure and mechanical properties of the components fabricated by WAAM, also clarified and compared the mechanical properties of 304L stainless steel components fabricated by different AM methods and forged standard. After analysing the result and summarizing the conclusion, it's found that the mechanical properties of the parts can satisfy the requirements of use.

\section{Experimental details}

The experiment of 304L stainless steel was based on the experimental setup developed for wire arc additive manufacturing (Figure 1). As the Figure 1(a) shows, the developed WAAM system mainly consists of a wire feeder, a computer, the working chamber and the GTAW equipment. The TIG welding torch is attached to a threeaxis machine. The melting source used to melt the wire is provided by the GTAW machine (Dynasty 350; Miller, USA). The diameter of the $304 \mathrm{~L}$ wire used in the experiment is $1.2 \mathrm{~mm}$. The entire studio environment is filled with $99.99 \%$ of the argon in case the parts are oxidized during the manufacturing process. The physical picture of the WAAM system is shown on the Figure 1(b).

The substrate used in the experiment is also $304 \mathrm{~L}$ stainless steel, whose size is $150 \mathrm{~mm} * 150 \mathrm{~mm} * 5 \mathrm{~mm}$. The moving path of torch and the wire are determined by the control centre. As the material overlaps the layers on the substrate, a box-like structure with 4 walls is formed, as shown in Figure 2(a). In order to get the mechanical properties of the stainless steel, six tensile specimens were cut by wire-electrode cutting. Respectively, the first 3 specimens who is parallel to the substrate are remarked as $\mathrm{T} 1, \mathrm{~T} 2, \mathrm{~T} 3$, and the 3 specimens who is perpendicular to the substrate are remarked as L1, L2, L3, as the Figure 2(b) shows. The tensile specimens were tested on an electronic universal material testing machine (Instron 5966, Instron, Germany) at room temperature. The tensile specimen is cut in accordance with the size of Figure 3(a). Figure 3(b) shows the standard tensile specimen and the fractured tensile specimens after tensile test. Obviously, it can found that the specimen is stretched a lot compared to the standard specimen.

Table 1. Process parameters used in WAAM

\begin{tabular}{|c|c|c|}
\hline $\begin{array}{c}\text { Process } \\
\text { parameters }\end{array}$ & Unit & Values \\
\hline Arc current & $\mathrm{A}$ & 280 \\
\hline Peak current & Ratio & $30 \%$ \\
\hline $\begin{array}{c}\text { Deposition } \\
\text { speed }\end{array}$ & $\mathrm{mm} / \mathrm{min}$ & 175 \\
\hline $\begin{array}{c}\text { Wire-feed } \\
\text { speed }\end{array}$ & $\mathrm{cm} / \mathrm{min}$ & 100 \\
\hline $\begin{array}{c}\text { Pulse } \\
\text { frequency }\end{array}$ & $\mathrm{Hz}$ & 1.5 \\
\hline
\end{tabular}


Before observing the microstructure and macrostructure of the samples, several steps need to be conducted. First, the SiC sandpaper with different fineness were used to polish the sample until the metal surface becomes flat. Then, the sample is polished by a

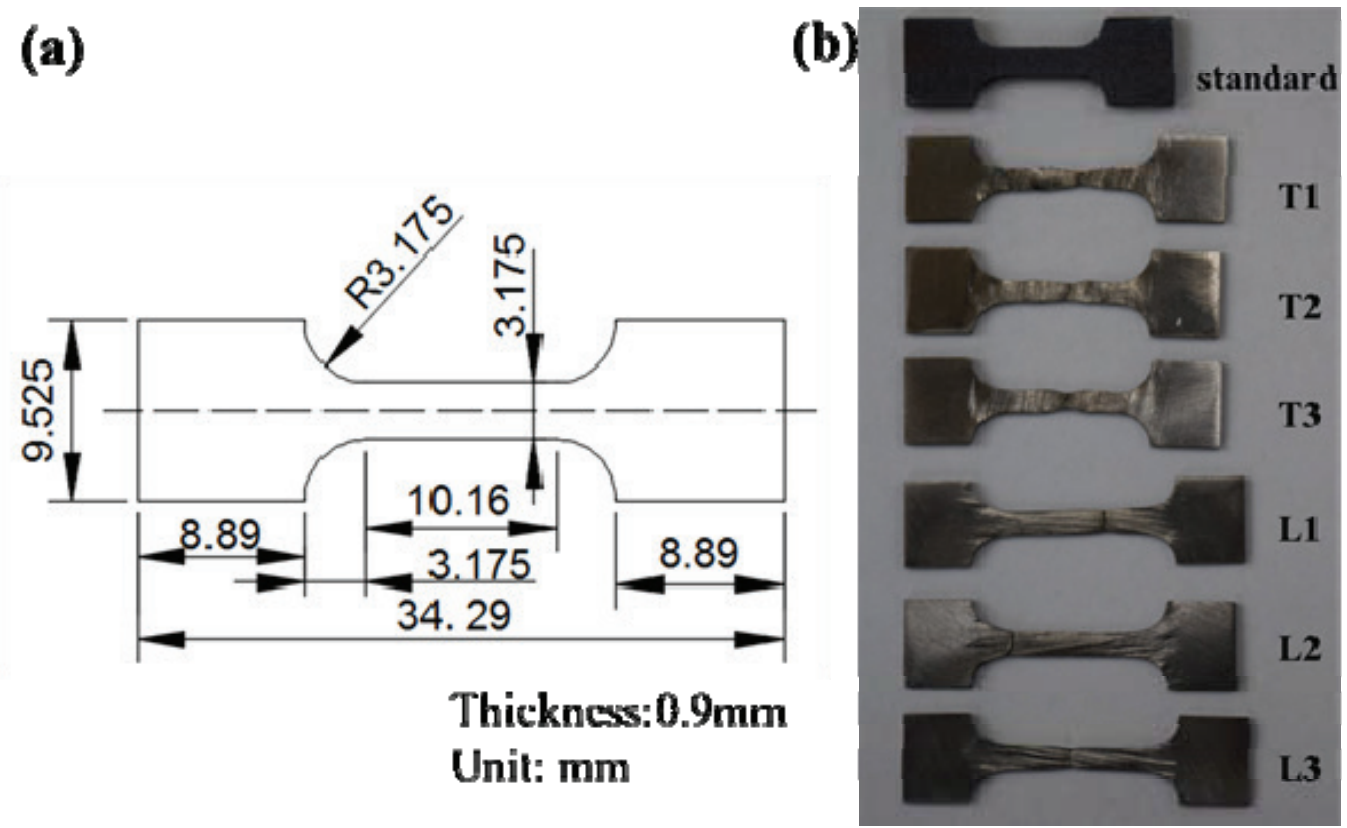

Figure 3. (a) Dimensions of tensile specimens and (b) Comparison of cracked tensile specimens and standard tensile specimens

polishing machine to get a mirror surface. Finally, according to the ratio of $1: 3: 2$, respectively taking $10 \mathrm{ml}$ HNO3 (nitric acid), 30ml HCL (hydrochloric acid), 20ml glycerol to configure aqua regia, then etching the metallographic sample about 40 seconds. In this way, the microstructures of samples were characterized by optical microscopy (Leica DM4000M; Leica, Germany)

The processing parameters used in the experiment are given in Table 1.

\section{Results and Discussions}

\subsection{Morphology and microstructure}

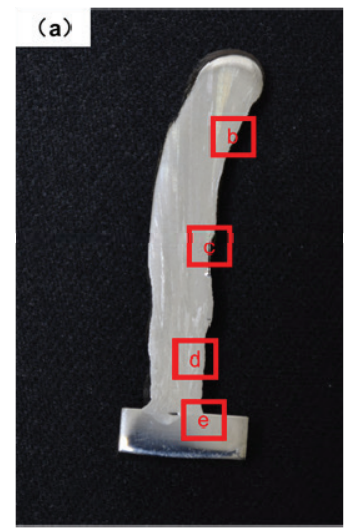

In order to analyse the structure of the parts fabricated by WAAM, the surface morphology and microstructure characteristics of the parts were described by observing the metallographic samples. As shown in Figure 4(a), the columnar grains on the surface were clearly visible after chemical etching. From the top of the part to the substrate, four regions (b, c, d, e) were selected to observe their characteristics of the microstructure. On the whole, the growth of the whole organization grows in a dendritic way. The Figure. 4(e) shows the junction of the substrate and the part, and the substrate is the rolled 304L stainless steel plate. Through the rolling method, the casting structure of the ingot is destroyed. The grains of the steel are refined and the defects of the microstructure are eliminated. Therefore, the rolled steel obtains a perfect structure with a dense organization. The first few layers

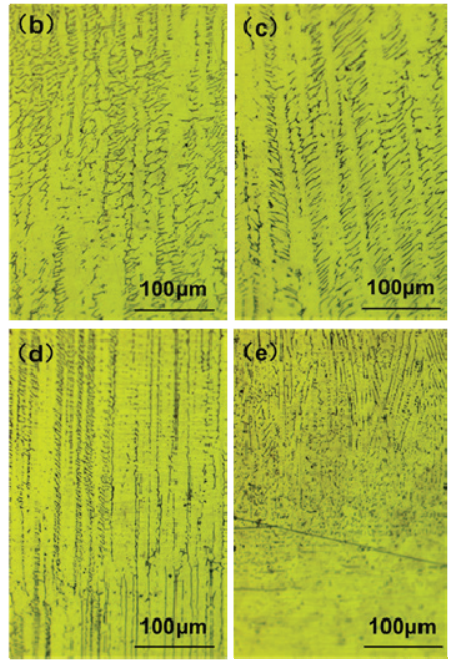

Figure 4. (a)Overview of the metallographic sample and (b, c, d, e) optical micrographs of the metallographic sample in different areas. 
which is contact with the substrate are full of fine and messy dendrites. The reason why the dendrite orientation is complex and irregular is that the substrate temperature is relatively low when the parts just started melding which leads to the speeding up the cooling rate and producing more nucleation. This area can be called the transition zone. The primary dendrite arm space of this area is $4.94 \mu \mathrm{m}$ and the secondary dendrite arm space is $3.52 \mu \mathrm{m}$. In Figure 4(d), the dendrites have a clear growth direction, showing a small columnar grain. Due to the process of grain growth is a kind of preferential growth process, the growth direction along the temperature gradient becomes obvious. In this area, the primary dendrite arm space is $8.23 \mu \mathrm{m}$, secondary dendrite arm space is $4.98 \mu \mathrm{m}$. As shown in Figure 4(c) and Figure 4(b), steady-state coarse austenitic columnar grains have been formed and secondary dendrites have appeared. The primary arm dendrite space is $13.59 \mu \mathrm{m}$ and $15.44 \mu \mathrm{m}$ respectively, and the secondary dendritic arm space is $8.93 \mu \mathrm{m}$ and $7.14 \mu \mathrm{m}$ respectively. Fig 5 shows the different dendritic spacing on the b, c, d, e areas.

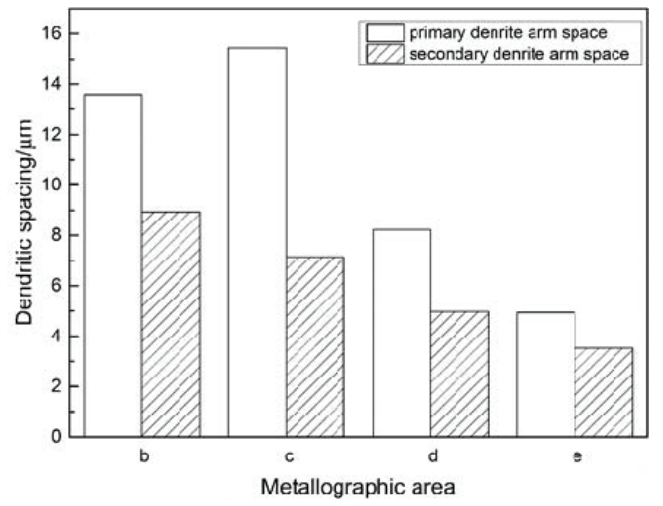

Figure 5. The different dendritic spacing on b, c, d, e areas.

From the region (e) of the substrate to the region (b) of the top, with the number of layers increasing, the heat input becomes larger and the cooling rate decreases. And the growth tendency of the grains becomes stable and single. The distribution of the organization can be seen from the schematic of Figure 6. This schematic reflects the distribution of metallographic images. The pulsed arc causes a number of molten pools formed on the substrate. When the liquid metal contacts with the low temperature substrate, the intense cooling of the liquid results in a great degree of undercooling, and the substrate with room temperature promote the non-spontaneous nucleation. So the liquid metal of this part produces a large number of nuclei and forms a layer of fine grain area manifesting as fine and messy dendrites. With the number of layers gradually increasing, the rate of cooling decreases and the direction of heat dissipation becomes apparent along the direction of accumulation of the material, the columnar grains appear parallel to each other, and the primary dendrite arm space and the secondary dendrite arm space become wider as well. At the middle position of the part, the large columnar grains appear, and the metallographic organization becomes stable. Not only the primary dendritic space and the secondary dendrites space become larger, even some three dendrites appear. This also shows that the cooling rate is pretty slow at this time. Because the direction of heat dissipation is also perpendicular to the direction of the substrate, the growth direction of the grains is along the gradient of the temperature.

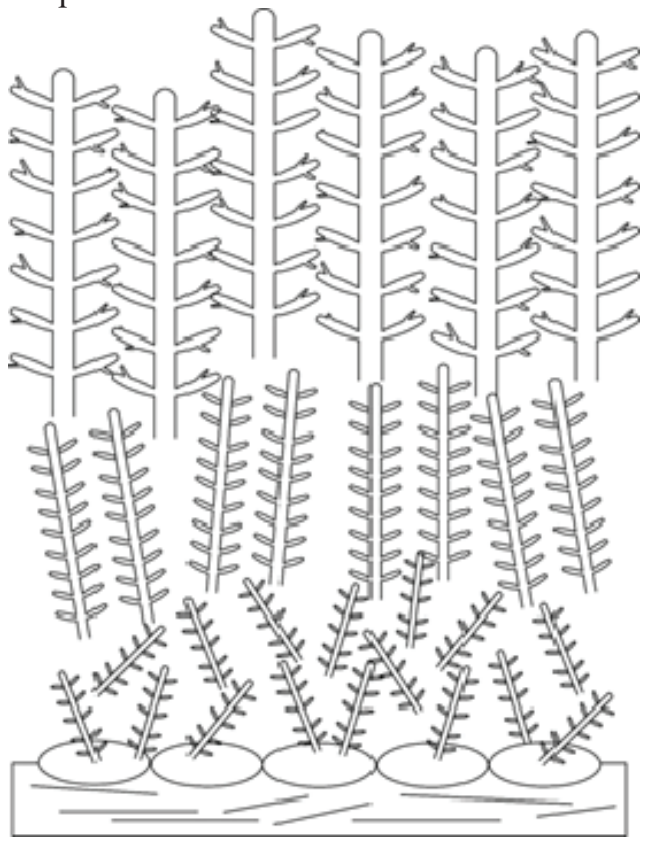

Figure 6. Schematic view of epitaxial grow of dendrites on the part fabricated by WAAM

\subsection{Tensile strength}

In order to obtain the mechanical properties of the parts, a tensile test was conducted to investigate the strength and elongation of the parts. Six tensile samples were fabricated to complete the test, including the transverse specimens T1, T2, T3 and the longitudinal specimens L1, L2, L3.

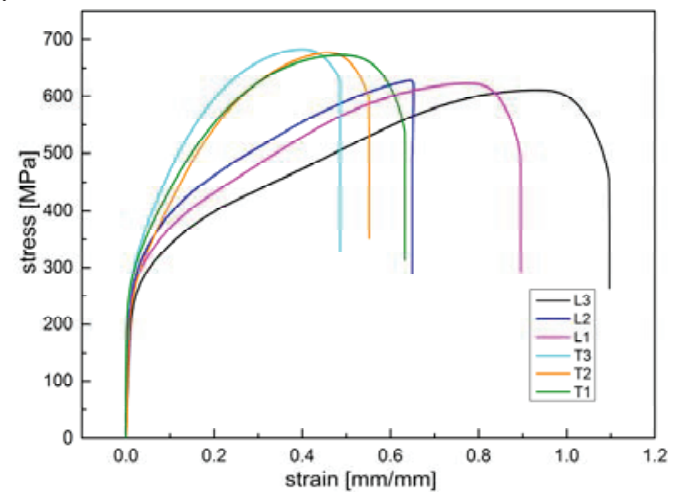

Figure 7. Stress-strain curves of the tensile specimens in longitudinal direction and transverse direction.

In general, 304L austenitic stainless steels have high ductility, low yield strength and relatively high ultimate tensile strength. Figure 7 shows the stress-strain curve of the tensile specimens. Their tensile strength, ultimate strength and elongation were measured and averaged over several samples. Overall, it is found that the performance of the series $\mathrm{L}$ is better than the series $\mathrm{T}$. Three kinds of phenomenon were mainly found in this research. Firstly, it was clear that the parts made of 
WAAM exist anisotropy in the presence of mechanical properties. As the Table 2 shows, in this study, the tensile strength and ultimate strength of transverse specimens were larger than the longitudinal specimens fabricated by WAAM, and the elongation of the transverse samples was far lower than the longitudinal samples. Besides, in the previous study, some parts produced by the methods of other additive manufacturing exist the anisotropy problem as well[13, 14]. A summary of mechanical properties of austenitic steel parts made by various AM methods, compared with forged plate[7, 16], is given in Table 2. Coincidentally, the previous study also found that the tensile strength and ultimate tensile strength of the transverse samples are greater than the longitudinal samples, and the elongation is also worse than the longitudinal samples, as the Table 2 shows. There is no doubt that this performance confirms the existence of anisotropy in the additive manufacturing methods. Compared with LMD and SLM, although these methods all belong to additive methods, the heat input of WAAM is larger than others and its cooling rate is lower. Therefore, the microstructure organization of the WAAM is coarse, which is different from the other AM ways with the laser resources. As a consequence, just like the Table 2 shows, the WAAM exhibits a lower yield strength and a lower ultimate tensile strength, but a good ductility compared with SLM and LMD. Finally, Table 2 also shows the standard values of the mechanical properties of forged 304L stainless steel. It is clear that the mechanical properties of the parts fabricated by WAAM are basically the same as forged plate. And the additive manufacturing method can effectively save the processing time cost, reduce the amount of post-processing time, also can reduce the waste of materials and improve the utilization of materials. So WAAM could have a great application prospect in the 304L large structural parts used in nuclear power.

Anisotropy is also found in previous studies, K.Zhang et al.[17] found the microstructure of SS316 components made by AM are anisotropic, they hold the view that the microstructural grains and ferrite dendrites are preferentially orientated along the highest thermal gradient, Zhuqing Wang et al.[13] fabricated the 304L parts by DED, they said that the long and narrow columnar grains growing along the build direction result in anisotropic tensile elongation properties. Combined with previous studies, the formation mechanism of anisotropic of 304L is discussed in the next.

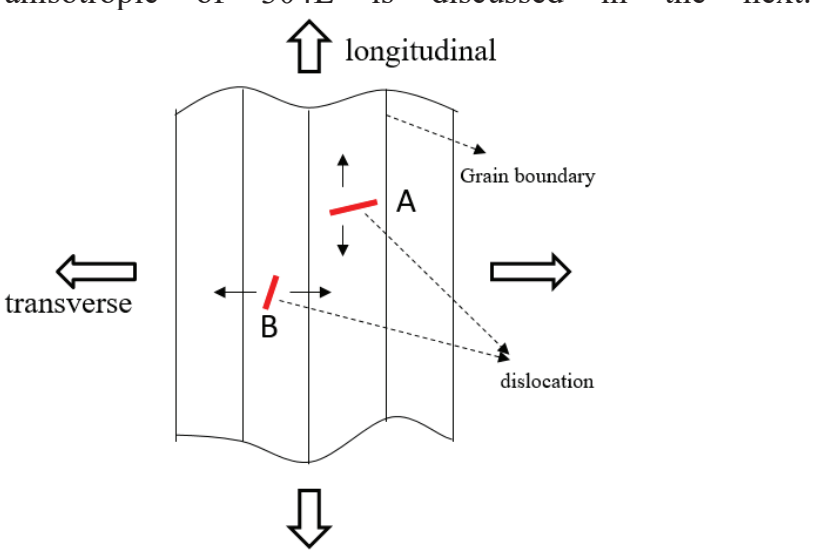

Figure 8. Schematic of grain boundary strengthening

The mechanical properties of materials are closely related to grain boundaries. Grain boundary is the interface between two adjacent grains with different space orientation, which will directly affect dislocation motion. The finer the grains are, the more grain boundaries exist, and the dislocation movement will get more resistance. Once meeting the grain boundary, the grain slippage will be end, that is to say, it is difficult to pass the boundary for the slippage. At the same time, dislocations pile up at the grain boundary, which is easy to produce stress concentration and produce cracks later. As a consequence, the plasticity is reducing.

The position of the tensile specimens used in the experiment is located in the middle section of the whole part. In this position, the microstructure is an elongated dendritic structure, and the growth direction is stable. It means that the direction is perpendicular to the substrate. As the Figure 8 shows, the transverse and longitudinal mean the direction of the tensile axis of the tensile specimens. When the loading direction is along the transverse direction, the dislocation $\mathrm{B}$ will appear. When the dislocation $\mathrm{B}$ slips along the transverse tensile direction, it's found that there is a grain boundary as a barrier. Because of the grain boundary strengthening effect, the tensile strength of the transverse direction is relatively high. On the other hand, when dislocation A slips along the longitudinal tensile direction, it is not obstructed by the grain boundary, which leads to the lower tensile strength.

Table 2. Summary of mechanical properties of 304 and 304L stainless steel fabricated by additive manufacturing compared with forged properties.

\begin{tabular}{cccccc}
\hline $\begin{array}{c}\text { Additive } \\
\text { manufacturing }\end{array}$ & $\begin{array}{c}\text { Stainless steel } \\
\text { alloy }\end{array}$ & Orientation & $\begin{array}{c}\text { Yield } \\
\text { strength } \\
(\mathrm{MPa})\end{array}$ & $\begin{array}{c}\text { Ultimate tensile } \\
\text { strength }(\mathrm{MPa})\end{array}$ & $\begin{array}{c}\text { Elongation } \\
(\%)\end{array}$ \\
\hline LMD $^{[13]}$ & 304L & Transverse & 337 & 609 & 48.2 \\
& & Longitudinal & 314 & 606 & 56.4 \\
SLM $^{[1]}$ & 304 & Transverse & 535 & 693 & 41.8 \\
& & Longitudinal & 455 & 580 & 57.5 \\
WAAM & 304L & Transverse & 235 & 678 & 55.6 \\
& & Longitudinal & 231 & 622 & 88.1 \\
Forged ${ }^{[18]}$ & 304L & & $\geq 170$ & $\geq 485$ & $\geq 40$ \\
\hline
\end{tabular}




\section{Conclusions}

In order to investigate the microstructure and tensile strength, a 304L stainless steel part with four walls were fabricated using wire arc additive manufacturing, and compared with other additive manufacturing methods and forged standard. The main conclusions from this study are as flows:

The microstructure of 304L stainless steel parts made by WAAM is filled with a large number of dendrites, and the growth direction is along the temperature gradient. From the first layer to the top, the morphology of the dendrites changes from fine and mess to large and stable, and the primary dendrite arm space changes from $4.94 \mu \mathrm{m}$ to $13.59 \mu \mathrm{m}$, the secondary dendrite arm space changes from $3.52 \mu \mathrm{m}$ to $8.93 \mu \mathrm{m}$.

Through the testing and analysis of mechanical properties, it is found that the strength and elongation were at forged level and the parts can satisfy the requirements of application. Like other additive manufacturing methods, the mechanical properties also exist the anisotropy, which attributes to the effect of the grain boundary strengthening is different in different directions.

\section{References}

1. Guan K, Wang Z, Gao M, et al. Materials \& Design. 50 581-586 (2013).

2. Girija S, Kamachi Mudali U. Corrosion Engineering, Science and Technology. 49 335-344 (2013).

3. Guo J, Zhou Y, Liu C, et al. Materials. 9823 (2016).

4. Lin C-M, Tsai H-L, Cheng C-D, et al. Engineering Failure Analysis. 21 9-20 (2012).

5. Frazier WE. Journal of Materials Engineering and Performance. 23 1917-1928 (2014).

6. Wang YD, Tang HB, Fang YL, et al. Materials Science and Engineering: A. 528 474-479 (2010).

7. Wang T, Zhu YY, Zhang SQ, et al. Journal of Alloys and Compounds. 632 505-513 (2015).

8. Wu Q, Ma Z, Chen G, et al. Journal of Manufacturing Processes. 27 198-206 (2017).

9. Shi X, Ma S, Liu C, et al. Materials Science and Engineering: A. 684 196-204 (2017).

10. Gong $\mathrm{X}$, Anderson T, Chou K. Manufacturing Review. 1:2 (2014).

11. Yilmaz O, Ugla AA. The International Journal of Advanced Manufacturing Technology. 89 13-25 (2016).

12. Abd-Elghany K, Bourell DL. Rapid Prototyping Journal. 18 420-428 (2012).

13. Wang Z, Palmer TA, Beese AM. Acta Materialia. 110 226-235 (2016).

14. Carroll BE, Palmer TA, Beese AM. Acta Materialia. 87 309-320 (2015).

15. Abe T, Sasahara H. Precision Engineering. 45 387395 (2016).
16. Wang F, Williams S, Rush M. The International Journal of Advanced Manufacturing Technology. 57 597-603 (2011).

17. Zhang K, Wang S, Liu W, et al. Materials \& Design. 55 104-119 (2014).

18. Standard Specification for Chromium and Chromium-Nickel Stainless Steel Plate, Sheet, and Strip for Pressure Vessels and for General Applications / Note: 2. revision 2004. 1. editorial change. Astm. 2001. 